obesity which may be more amenable to change, is a risk factor for obesity and chronic health conditions in adults.

Methods Socioeconomically disadvantaged children aged 4-7y and their parents in Beer-Sheva were the study population in an intervention trial. We excluded those who refused, children with any chronic disease, developmental problems, in a weight reduction treatment and children or parents with any psychiatric problem. We measured twice weight and height of children and mothers in the mornings before breakfast, with light clothing and without shoes. Other data were obtained by personal interviews. Smoking and maternal perception of the child's weight status will be examined using the baseline data.

Results Overall $18.5 \%$ and $11.3 \%$ of the children were overweight and obese, respectively. Overweight/obese (OWO) children were significantly taller, heavier and had more sedentary hours than nonOWO children. Mothers misclassified the child's weight status in $82.3 \%$ and $42.4 \%$ of OWO and non-OWO children, respectively $(p<0.001)$. In a multivariate logistic regression which included the child's sedentary hours, maternal education and weight status, poverty status, only misclassification of the child's weight status (OR 8.3, 2.7-25.9; $\mathrm{p}<0.001$ ) and current parental smoking (OR 4.2, $1.6-11.4 ; p=0.005)$ were significant risk factors for OWO in LSES pre-school children.

Conclusions Maternal misclassification of her child's weight status and parental smoking may be determinants of the development of childhood obesity among LSES children. These associations should be confirmed in prospective studies.

\section{P1-426 ASSESSING THE PSYCHOMETRICS AND THE ECOMETRICS MEASUREMENTS OF NEIGHBOURHOOD SCALES: SAÚDE EM BEAGÁ STUDY, BELO HORIZONTE, BRAZIL, 2008-2009}

doi:10.1136/jech.2011.142976g.16

\begin{abstract}
${ }^{1,2}$ A Friche, ${ }^{*}{ }^{3} \mathrm{~A}$ Diez-Roux, ${ }^{1,2} \mathrm{C}$ César, ${ }^{1,2}$ F Proietti, ${ }^{1,2} \mathrm{~W}$ Caiaffa. ${ }^{1}$ Graduate Program of Public Health, School of Medicina, Federal University of Minas Gerais, Belo Horizonte, Minas Gerias, Brazil; ${ }^{2}$ Observatory for Urban Health, Federal University of Minas Gerais, Belo Horizonte, Minas Gerias, Brazil; ${ }^{3}$ Center for Social Epidemiology and Population Health, School of Public Health, University of Michigan, Ann Arbor, Michigan, USA
\end{abstract}

Introduction Although relevant, specific instruments are necessary to better understand the relationship between features of neighbourhood and health events, very few studies have developed instruments to measure neighbourhood features in developing countries. Objective To develop valid and reliable measures of neighbourhood context useful in a Latin American urban context; assess their psychometrics and ecometrics properties and examine individual and neighbourhood-level predictors of these measures.

Methods We analysed data from a multistage (census tracts, households, and residents) household survey (2008-2009) conducted in Belo Horizonte City by the Observatory for Urban Health. One adult in each household was selected to answer the questionnaire that was composed of six domains. Neighbourhood was defined as census tract. Scales to represent different dimensions of neighbourhood were created using individual responses. Internal consistency was evaluated by Cronbach's $\alpha$ and three-level multilevel models were used to evaluate each scale.

Results 4048 survey respondents represented 149 census tracts. We assessed nine neighbourhood environment dimensions: Public Services (8 items), Aesthetic Quality (4 items), Walking Environment (7 items), Violence (6 items), Social Cohesion (6 items), Activities with Neighbours (11 items), Neighbourhood Physical Disorders (8 items), Neighbourhood Social Disorders (5 items) and Neighbourhood Problems (16 items). Cronbach's $\alpha$ coefficient ranged from 0.53 to 0.83 , intraneighborhood correlation ranged
$0.02-0.53$ and neighbourhood reliability were $0.76-0.99$. Most scales were associated with individual and neighbourhood predictors.

Conclusion These findings illustrated the measurement properties of neighbourhood-level constructs can be measured reliably, confirming their use in multilevel analysis to assess the neighbourhood effects on health outcomes.

\section{P1-427 ASSOCIATIONS OF MATERNAL WEIGHT GAIN IN PREGNANCY WITH OFFSPRING COGNITION THROUGHOUT CHILDHOOD AND ADOLESCENCE}

doi:10.1136/jech.2011.142976g.17

S Gage, ${ }^{*}$ D Lawlor, K Tilling, A M Tolppanen, A Fraser. University of Bristol, Bristol, UK

Introduction Basic science evidence suggests that gestational weight gain (GWG) may influence offspring cognitive development. However, this relationship has not been investigated in human population studies.

Methods Data from the ALSPAC, a UK prospective pregnancy cohort were used. GWG was expressed using 2009 IOM categories of GWG and estimates from random effect linear spline models (median number of measures per woman: 10 IOR: 8-11). Outcomes were school entry assessment score (SEA, age 4, N=5832), Wisc-III assessed IO (age 8, N=5191) and GCSE results (age 15, N=7339).

Results Offspring of women who gained less than the 2009 IOM recommended GWG had a -0.075 SD lower mean SEA score $(95 \% \mathrm{CI}$ -0.127 to -0.023 ) compared with women who gained as recommended, even when adjusting for potential confounders including maternal education. Greater prepregnancy weight was inversely associated with all cognition measures. For example, mean difference in IQ per $1 \mathrm{~kg}$ increase in pre-pregnancy weight $=-0.004 \mathrm{SD}$ $(-0.006,-0.002)$. GWG in early pregnancy $(0-18$ weeks $)$ and in mid-pregnancy (19-28 weeks) were positively associated with SEA and IO but not with GCSE results. GWG in late pregnancy (29 + weeks) was positively associated with higher SEA scores $(0.208 S D ; 0.716,0.261)$ and GCSE results $(\mathrm{OR}=1.35 ; 1.26,1.46)$, with the latter not fully mediated by the association with SEA.

Conclusions Findings support a positive association between GWG, particularly in late gestation, and offspring cognitive development, which has lasting effects on school attainment at age 16 years. However this could still be due to residuals confounding.

\section{P1-428 PREVALENCE AND RISK FACTORS OF HEPATITIS C VIRUS INFECTION AMONG POLISH NURSES AND MIDWIVES}

doi:10.1136/jech.2011.142976g.18

${ }^{1}$ M Ganczak, ${ }^{* 2}$ M Korzen, ${ }^{1}$ Z Szych. ${ }^{1}$ Pomeranian Medical University, Szczecin, Poland; ${ }^{2}$ Westpomeranian University of Technology, Szczecin, Poland

Surgical and midwifery staff are at risk of acquiring occupational blood-borne infections. Controversial results have been reported about HCV. Objective of this cross-sectional sero-epidemiological study was to assess the prevalence of HCV in nurses from surgical/ gynaecological wards of 16 randomly selected hospitals in West Pomeranian region of Poland and to compare it with other groups: consecutive female patients from the same hospital wards and female blood donation candidates from Regional Center for Blood Donation. Method: Serum samples collected from 414 healthcare employees and 1118 female patients have been tested by ELISA between February 2008 and June 2009 and confirmed by RIBA. Results The seroprevalence in staff members was $1.4 \%(95 \% \mathrm{CI}$ $0.7 \%$ to $3.1 \%$ ). Personnel's sero-positive status was predominantly discovered during our occasional screening. A stepwise multivariate 